\section{Band 10, Heft 4, August 2003}

\section{Editorial}

176 Universitäre Forschung in Naturheilkunde und Komplementärmedizin - Perspektiven und Standortbestimmung Melchart, D. (München)

\section{Originalarbeiten}

179 Akupunktur-randomisierte Studien (ART) bei Patienten mit Migräne und Spannungskopfschmerzen - Design und Protokolle

Melchart, D., Linde, K., Streng, A., Reitmayr, S., Hoppe, A. (München); Brinkhaus, B., Becker-Witt, C. (Berlin); Wagenpfeil, S., Pfaffenrath, V., Hammes, M. (München); Willich, S.N. (Berlin); Weidenhammer, W. (München)

185 Akupunktur-randomisierte Studien (ART) bei Patienten mit chronischen Schmerzen im Bereich der Lendenwirbelsäule und Gonarthrose - Design und Protokolle

Brinkhaus, B., Becker-Witt, C., Jena, S. (Berlin); Linde, K., Streng, A., Wagenpfeil, S., Irnich, D., Hummelsberger, J., Melchart, D. (München); Willich, S.N. (Berlin)

192 Verschränkung: Ein theoretisches Modell für die Homöopathie als Beispiel für generalisierte Verschränkung als Konsequenz aus einer verallgemeinerten Quantentheorie Walach, H. (Freiburg i. Br.)

\section{Leitthema: Gesundheit}

Essay

201 Eine kurze Einführung in die Erweiterte Netzwerk- und Verschränkungstheorie als Theorie des Heilens und ihre empirischen Vorhersagen Hyland, M.E. (Plymouth, UK)
Vol. 10, Issue 4, August 2003

Editorial

176 University Research in Natural and Complementary Medicine - Prospects and State of the Art Melchart, D. (München)

Original Articles

179 Acupuncture Randomized Trials (ART) in Patients with Migraine or Tension-Type Headache - Design and Protocols

Melchart, D., Linde, K., Streng, A., Reitmayr, S., Hoppe, A. (München); Brinkhaus, B., Becker-Witt, C. (Berlin); Wagenpfeil, S., Pfaffenrath, V., Hammes, M. (München); Willich, S.N. (Berlin); Weidenhammer, W. (München)

185 Acupuncture Randomized Trials (ART) in Patients with Chronic Low Back Pain and Osteoarthritis of the Knee Design and Protocols

Brinkhaus, B., Becker-Witt, C., Jena, S. (Berlin); Linde, K., Streng, A., Wagenpfeil, S., Irnich, D., Hummelsberger, J., Melchart, D. (München); Willich, S.N. (Berlin)

192 Entanglement Model of Homeopathy as an Example of Generalized Entanglement Predicted by Weak Quantum Theory Walach, H. (Freiburg i. Br.)

Focus: Health

Essay

201 A Brief Guide to Extended Network Entanglement Theory as a Theory of Healing and Its Empirical Predictions Hyland, M.E. (Plymouth, UK)

\section{KARGER}

Fax +49761 4520714

E-mail Information@Karger.de www.karger.com
(C) 2003 S. Karger GmbH, Freiburg 


\section{Band 10, Heft 4, August 2003}

Proceedings

207 4. Symposium zur Ratio und Plausibilität in der Naturheilkunde. «Migräne» - Persönlichkeitsstigma oder behandelbares Leiden? 7. Dezember 2002, Friedrich-Schiller-Universität Jena Gast-Herausgeber: Uhlemann, C. (Jena)

\section{Journal Club}

217 «Ionisierte» Armbänder für muskuloskelettale Schmerzen entzaubert

218 Entfernung von Zahn-Amalgam: Wackelige Ergebnisse durch Überinterpretation der Daten

219 Untersuchung homöopathischer Hochpotenzen mittels «gas discharge visualization»: Gleichung mit zwei Unbekannten

222 Nackenschmerzen: Manuelle Verfahren sind der Kunst des Hausarztes überlegen!

224 Nackenschmerzen: Manualtherapie kosteneffektiv

Gesellschaftsmitteilungen

225 SMGP Schweizerische Medizinische Gesellschaft für Phytotherapie

227 Buchbesprechungen

230 Industrieforum

234 Tagungen und Kongresse

232 Impressum

U2 Hinweise für Autoren (2. Umschlagseite)

Einen Ausblick auf den Inhalt der kommenden Hefte finden Sie auf Seite 236.
Vol. 10, Issue 4, August 2003

Proceedings

207 4th Symposium to Reason and Plausibility in Natural Medicine. 'Migraine' - Stigma of the Personality or Treatable Suffering? December 7, 2002, Friedrich Schiller University Jena

Guest Editor: Uhlemann, C. (Jena)

\section{Journal Club}

217 Spell Broken on 'Ionized' Wrist Bracelets for Musculoskeletal Pain

218 Dental Amalgam Removal: Overinterpretation of Data Precludes Reliable Conclusions

219 Gas Discharge Visualization Evaluation of Ultramolecular Doses of Homeopathic Remedies: One Equation with Two Unknown Variables

222 Neck Pain: Manual Therapies Score Better than GP Care!

224 Neck Pain: Manual Therapy Cost-Effective

Society Bulletins

225 SMGP Schweizerische Medizinische Gesellschaft für Phytotherapie

227 Book Reviews

230 Industrial Forum

234 Meetings and Conferences

232 Imprint

U2 Guidelines for Authors (Inside front cover)

Prospects for contents of the next issues are given on page 236. 